\title{
Correlation between changes in quality of life and symptomatic improvement in Chinese patients switched from typical antipsychotics to olanzapine
}

This article was published in the following Dove Press journal:

Neuropsychiatric Disease and Treatment

19 January 2015

Number of times this article has been viewed

\section{William Montgomery'}

Zbigniew Kadziola ${ }^{2}$

Wenye $\mathrm{Ye}^{3}$

Hai Bo Xue ${ }^{4}$

Li Liu ${ }^{4}$

Tamás Treuer ${ }^{5}$

'Global Patient Outcomes and Real World Evidence, Eli Lilly Australia Pty Ltd, West Ryde, NSW, Australia; ${ }^{2}$ Real World Analytics Capabilities, Eli Lilly GmbH,Vienna, Austria; ${ }^{3}$ Real World Analytics, Eli Lilly and Company, Indianapolis, IN, USA; ' 4 Lilly Suzhou Pharmaceutical Company, Ltd, Shanghai, People's Republic of China; ${ }^{5}$ Neuroscience Research, Eli Lilly and Company, Budapest, Hungary
Correspondence: William Montgomery Global Patient Outcomes and Real World Evidence, Eli Lilly Australia Pty Ltd, I I Wharf Rd, West Ryde, NSW 2I I4, Australia

Tel +6I 293254335

Fax +6I 293254334

Email montgomery_bill@lilly.com
Purpose: The aim of this study was to investigate the correlation between changes in symptoms and changes in self-reported quality of life among Chinese patients with schizophrenia who were switched from a typical antipsychotic to olanzapine during usual outpatient care.

Patients and methods: This post hoc analysis was conducted using data from the Chinese subgroup ( $\mathrm{n}=475$ ) of a multicountry, 12-month, prospective, noninterventional, observational study. The primary publication previously reported the efficacy, safety, and quality of life among patients who switched from a typical antipsychotic to olanzapine. Patients with schizophrenia were included if their symptoms were inadequately controlled with a typical antipsychotic and they were switched to olanzapine. Symptom severity was measured using the Brief Psychiatric Rating Scale (BPRS) and the Clinical Global Impressions-Severity scale (CGI-S). HealthRelated Quality of Life (HRQOL) was assessed using the World Health Organization Quality of Life-Abbreviated (WHOQOL-BREF). Paired $t$-tests were performed to assess changes from baseline to endpoint. Pearson's correlation coefficients $(r)$ were used to assess the correlations between change in symptoms (BPRS and CGI-S scores) and change in HRQOL (WHOQOLBREF scores).

Results: Symptoms and HRQOL both improved significantly over the 12 months of treatment $(P<0.001)$. Significant correlations were observed between changes from baseline to end of study on the BPRS and the CGI-S and each of the WHOQOL-BREF four domain scores and two overall quality-of-life questions. The correlation coefficients ranged from $r=-0.45$ to $r=-0.53$ for the BPRS and WHOQOL-BREF. The correlation coefficients were slightly smaller between the CGI-S and WHOQOL-BREF, ranging from $r=-0.33$ to $r=-0.40$.

Conclusion: For patients with schizophrenia, assessing quality of life has the potential to add valuable information to the clinical assessment that takes into account the patient's own perspective of well-being.

Keywords: data correlation, olanzapine, quality of life, schizophrenia, signs and symptoms

\section{Introduction}

Schizophrenia is a chronic and highly debilitating mental illness characterized by impaired thinking, feeling, behavior, and functioning. Because of the broad spectrum of impairment, many individuals with this disorder are unable to gain employment or maintain a marriage. ${ }^{1,2}$ According to guidelines for the treatment of patients with schizophrenia, the goals for treatment should include reducing or eliminating symptoms, improving adaptive functioning and quality of life to the fullest extent, and promoting and sustaining functioning to the highest degree possible. ${ }^{3}$ Beyond these general areas of focus for treatment, identifying specific treatment targets and using a range of outcome measures to assess treatment progress are also important. 
Antipsychotic medications are the foundation of the treatment for people with schizophrenia. They are recommended for use in acute psychotic episodes and for maintenance treatment to prevent future relapses. ${ }^{3}$ Adjunctive medications are also commonly prescribed for comorbid conditions. Although significant progress has been made in the advancement of medications used to treat schizophrenia, research in this area has often focused on the effectiveness of a given treatment in reducing clinical symptoms and the assessment of treatment-related adverse events. Measuring the degree of change in clinical symptoms and determining the relative safety of a therapeutic intervention are both important aspects to consider, but focusing more broadly on outcomes might help inform treatment decisions and facilitate the achievement of treatment goals. If one of the main goals of treatment is to improve adaptive function and quality of life, then determining the effectiveness or progress of treatment should include measures that capture quality of life. Assessing quality of life would provide complementary information and allow for a multidimensional approach to assessing outcomes. ${ }^{4}$

The World Health Organization (WHO) defines healthrelated quality of life (HRQOL) as an:

individual's perception of their position in life in the context of the culture and value systems in which they live and in relation to their goals, expectations, standards and concerns. It is a broad ranging concept affected in a complex way by the person's physical health, psychological state, personal beliefs, social relationships and their relationship to salient features of their environment. ${ }^{5}$

In schizophrenia, HRQOL has been identified as an important outcome of treatment. ${ }^{6-9}$ Although some have expressed concern about the validity of asking patients with psychosis to self-report HRQOL, ${ }^{7}$ a number of studies have shown this to be feasible, and a number of instruments used to assess HRQOL have demonstrated sensitivity to the status of patients with psychotic disorders. ${ }^{4}$ More recently, there has been growth in the number of publications about the assessment of HRQOL in schizophrenia. ${ }^{7}$ However, beyond its inclusion in clinical studies, assessing HRQOL in clinical practice remains underused,$^{10}$ and concerns continue to be expressed about the lack of effect of quality-of-life assessment on clinical care and health policy decision-making. ${ }^{?}$

Although some research in Western countries has examined the relationship between HRQOL and clinical outcomes in schizophrenia, ${ }^{11-14}$ there are fewer data available from Asia. The objective of this study was to investigate the correlation between improvements in physician-rated symptoms and changes in patient-reported quality of life among Chinese patients with schizophrenia who were switched from a typical antipsychotic to olanzapine during usual outpatient care.

\section{Methods Study design}

This was a post hoc analysis conducted using data from the Chinese subgroup of a multicountry, 12-month, prospective, noninterventional, observational study in an Asian outpatient population. The study had five visits (baseline, week 4 , week 8 , week 26 , and week 52 ). The primary manuscript reported the efficacy, safety, and quality-of-life changes among patients who switched from a typical antipsychotic to olanzapine. ${ }^{15}$ Data for the original study were collected from 64 investigator sites across the People's Republic of China, Hong Kong, the Philippines, South Korea, and Taiwan. The study began in May 2002 and was completed in September 2004. The study was carried out in accordance with the ethics and regulatory requirements for each region. Informed consent was provided by all participants, as determined by local regulations.

\section{Patients}

Patients met criteria for inclusion in the study if their treating physician diagnosed them with schizophrenia, according to the Diagnostic and Statistical Manual of Mental Disorders, Fourth Edition, ${ }^{16}$ or the International Classification of Diseases, Tenth Revision; ${ }^{17}$ if it was determined that their symptoms were inadequately controlled on a typical antipsychotic medication; and if they switched to the atypical antipsychotic olanzapine, at the physician's and patient's discretion. ${ }^{15}$ In addition, patients were required to be between 18 and 60 years of age and to have presented during the normal course of care. Patients were excluded if they had used olanzapine (or any other atypical antipsychotic) during the 6 months before enrollment or were pregnant.

To ensure the unobtrusive observation of usual clinical care, participating psychiatrists were asked to manage study patients per their usual practice. Patients were prescribed olanzapine within the recommended dose range, as determined by the psychiatrist. However, if patients stopped taking olanzapine, they were discontinued from the study. Concomitant medications, including other atypical or typical antipsychotics, were allowed.

\section{Measures}

Symptom severity, behavior, and functioning were measured using the Brief Psychiatric Rating Scale (BPRS) ${ }^{18}$ and 
the Clinical Global Impressions-Severity scale (CGI-S). ${ }^{19}$ The BPRS is composed of 24 questions and is used as part of a clinical interview to assess the positive, negative, and affective symptoms associated with schizophrenia. Each question is rated on a scale ranging from 1 (not present) to 7 (extremely severe). The CGI-S rating is based on symptoms, behavior, and functioning over the last 7 days, both reported and observed. It is rated on a 7 point scale ranging from 1 (normal) to 7 (among the most extremely ill patients).

HRQOL was measured using the World Health Organization Quality of Life-Abbreviated (WHOQOL-BREF) ${ }^{20}$ The WHOQOL-BREF is an abbreviated version of the World Health Organization Quality of Life-100 (WHOQOL-100) questionnaire, designed to assess the patient's perception regarding their well-being. It consists of 26 patient self-rated questions that capture the intensity, frequency, or evaluation of selected attributes of HRQOL during the previous 2 weeks on a 5 point rating scale. Four domain scores can be obtained from the 26 items: physical health, mental health, social relationships, and environment. Two questions measure the patient's overall quality of life, using a 5 point rating scale of very poor, poor, neither poor nor good, good, and very good: How would you rate your quality of life? and, How satisfied are you with your health? All assessments were made at baseline and at weeks 4 ( \pm 1 week), 8 ( \pm 1 week), 26 ( \pm 2 weeks), and 52 ( \pm 2 weeks).

Mandarin versions of all measures were used in this study. For additional details, please refer to the primary publication. ${ }^{15}$

\section{Statistical analyses}

Paired $t$-tests were performed to analyze changes from baseline to endpoint, using last observation carried forward. Only patients with a baseline measure and at least one postbaseline measure were included in the baseline-to-endpoint analyses. Correlations were tested between the change from baseline to endpoint on the BPRS and CGI-S total scores and the WHOQOL-BREF four domain scores and two overall quality-of-life questions, using Pearson's correlation coefficient $(r)$. A $P$-value of $<0.05$ was considered statistically significant, noting that interpretation of results should be made cautiously because of the number of tests conducted. All analyses were completed using SAS, version 9.2 (SAS, Inc., Cary, NC, USA).

\section{Results}

\section{Participants}

In the parent study, a total of 1,272 Asian subjects with schizophrenia were enrolled, of whom five did not meet eligibility criteria. ${ }^{15}$ Of the remaining 1,267 patients, 475 were from the People's Republic of China and form the subgroup for this analysis. Baseline characteristics are presented in Table 1. There were relatively equal numbers of males and females, and most (94\%) of the patients had been previously receiving monotherapy with a typical antipsychotic. Of the 475 patients at baseline, 437 (92\%) completed the 52-week study and $38(8 \%)$ discontinued. At baseline, 60 (13\%) patients were taking an additional antipsychotic and 138 (29\%) were prescribed other concomitant psychotropic medications such as benzodiazepines and antidepressants. At the end of the study, 38 (8\%) of the patients were taking an additional antipsychotic and $33(7 \%)$ were prescribed other concomitant medications.

\section{Symptoms}

At the end of the study, there were statistically significant improvements in clinical symptoms, as measured by the BPRS and CGI-S, with a decrease in score indicating improvement (Figure 1). The mean change from baseline to endpoint for the BPRS total score at the end of the study was -32.4 (95\% confidence interval $[\mathrm{CI}],-33.8$ to -31.1 ), which represents an $86 \%$ reduction $(P<0.001)$. The mean change from baseline to endpoint for the CGI-S at the end of the study was $-3.3(95 \% \mathrm{CI},-3.4$ to -3.2$)$, which represents a $65 \%$ reduction $(P<0.001)$.

\section{Quality of life}

Quality of life improved significantly on each of the WHOQOL-BREF four domain scores, with an increase in

Table I Baseline characteristics

\begin{tabular}{|c|c|}
\hline Variable & Value \\
\hline Age, mean (range) & $28(18-60)$ \\
\hline \multicolumn{2}{|l|}{ Sex, n (\%) } \\
\hline Male & $244(49 \%)$ \\
\hline Female & $231(51 \%)$ \\
\hline \multicolumn{2}{|l|}{ Typical antipsychotic, $\mathrm{n}(\%)$} \\
\hline Monotherapy & $448(94 \%)$ \\
\hline Combination therapy & $27(6 \%)$ \\
\hline \multicolumn{2}{|c|}{ Most common prior antipsychotics, $\mathrm{n}(\%)$} \\
\hline Chlorpromazine & $166(35 \%)$ \\
\hline Perphenazine & $|2|(26 \%)$ \\
\hline Haloperidol & $87(18 \%)$ \\
\hline BPRS total, mean $(95 \% \mathrm{Cl})$ & $37.3(36.0-38.7)$ \\
\hline CGI-S, mean $(95 \% \mathrm{Cl})$ & $5.0(4.9-5.1)$ \\
\hline \multicolumn{2}{|c|}{ WHOQOL-BREF, mean $(95 \% \mathrm{Cl})$} \\
\hline Physical health & $42.7(4||-44.2)$. \\
\hline Psychological & $40.7(39.2-42.3)$ \\
\hline Social relationships & $37.4(35.9-39.0)$ \\
\hline Environment & $45.0(43.6-46.3)$ \\
\hline
\end{tabular}

Abbreviations: BPRS, Brief Psychiatric Rating Scale; CGI-S, Clinical Global Impressions-Severity Scale; Cl, confidence interval; WHOQOL-BREF, World Health Organization Quality of Life-Abbreviated. 


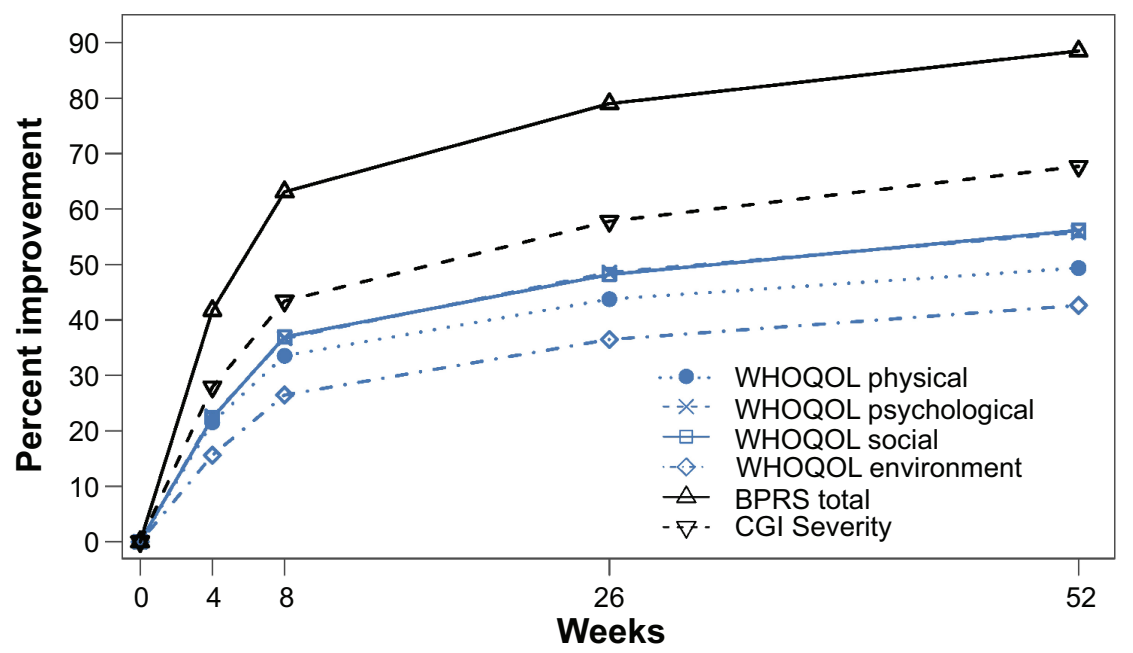

Figure I Percentage improvement from baseline in the BPRS, CGI-S, and WHOQOL-BREF four domain scores by visit.

Notes: The pattern of improvement in symptoms and HRQOL appeared to be similar over time. The greatest improvement occurred during the first 8 weeks. Abbreviations: BPRS, Brief Psychiatric Rating Scale; CGI, Clinical Global Impressions; HRQOL, Health-Related Quality of Life; WHOQOL-BREF, World Health Organization Quality of Life -Abbreviated.

score indicating improvement. Mean changes from baseline to end of study for each domain were physical health (25.2; 95\% CI, 23.4-26.9), psychological health (27.6; 95\% CI, 25.7-29.4), social relationships (25.9; 95\% CI, 23.9-27.9), and environment (23.3; 95\% CI, 21.6-25.0). The mean change from baseline for the WHOQOL-BREF overall quality of life question of "How would you rate your quality of life?" was 1.47 (95\% CI, 1.37-1.57). The mean change from baseline for the WHOQOL-BREF overall quality of life question of "How satisfied are you with your health?" was 1.35 (95\% CI, 1.25-1.45). The mean changes for all WHOQOL-BREF scores were statistically significant at the end of the study, as determined by paired $t$-tests $(P<0.001)$. The pattern of improvement over time for the WHOQOL-BREF domain, BPRS, and CGI scores was similar (Figure 1).

\section{Correlations between changes in symptoms and quality of life}

Statistically significant correlations were observed between changes from baseline to end of study on the BPRS and CGI-S and the WHOQOL-BREF overall quality-of-life question, "How would you rate your quality of life?" $(r=-0.46$ $[P<0.001] ; r=-0.37[P<0.001]$, respectively). Similarly, the correlations between changes from baseline to end of study on the BPRS and CGI-S and the WHOQOL-BREF overall quality of life question of "How satisfied are you with your health?" ( $r=-0.46[P<0.001] ; r=-0.33[P<0.001]$, respectively). Statistically significant correlations were also observed between changes from baseline to end of study on the BPRS and CGI-S and each of the WHOQOL-BREF four domain scores (Figure 2).

\section{Discussion}

In this post hoc analysis of a 12-month, prospective, noninterventional, observational study in Chinese outpatients with schizophrenia, patients who were switched to olanzapine experienced statistically significant improvements in clinical symptoms across all physician-reported measures. Consistent with the changes in physician-reported clinical symptoms, improvements in HRQOL were also observed on the basis of patient-reported measures. As seen in Figure 1, changes in the BPRS, CGI-S, and WHOQOLBREF four domain scores showed a rapid improvement over the first 8 weeks, with continued improvement until the end of the study. The early improvement in symptoms and patient-perceived HRQOL observed in this study is consistent with results from another study that found that patients who were inadequately controlled on a different oral antipsychotic medication and switched to olanzapine showed a significant improvement in subjective well-being after 1 week. ${ }^{21}$

The changes in clinical symptoms were correlated with changes in HRQOL. The correlation coefficients between the BPRS and CGI-S and WHOQOL-BREF scores ranged from $r=-0.45$ to $r=-0.53$ and $r=-0.33$ to $r=-0.40$, respectively. Correlations in this range are considered to be moderate to large. ${ }^{22}$ The negative correlation between clinical symptoms and the HRQOL scores indicates that as clinical symptoms improve (as reflected by lower scores on the BPRS and 


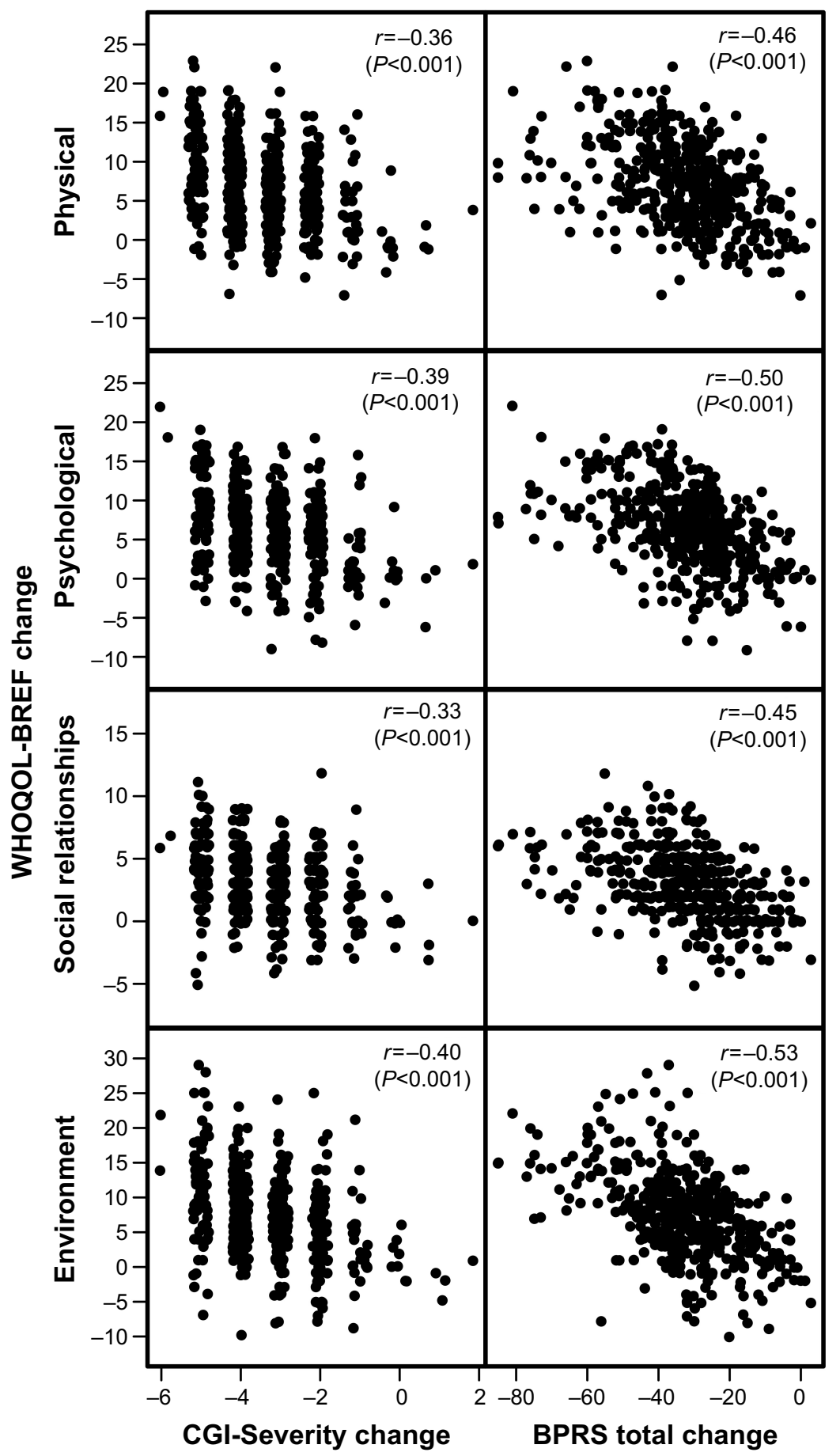

Figure 2 Scatter plots of change scores for BPRS and CGI-S versus WHOQOL-BREF domain scores.

Notes: Change scores were calculated on the basis of the last observation for each individual. Each plot is labeled with the Pearson correlation coefficient ( $r$ ). A small random variation was added to each point so the number of observations would be clear.

Abbreviations: BPRS, Brief Psychiatric Rating Scale; CGI, Clinical Global Impressions; WHOQOL-BREF, brief version of the World Health Organization Quality of Life Scale.

CGI-S), quality of life improves (as reflected by higher scores on the WHOQOL-BREF).

Although we are unaware of comparable research in the People's Republic of China, findings outside of the People's Republic of China are consistent with the findings of the current study. For example, a Canadian study also reported
HRQOL improvements in patients who were switched from typical antipsychotics to olanzapine. ${ }^{23}$ Similar to the moderate to large correlations between clinical symptoms and HRQOL found in this study, previous research reported meaningful correlations between the Quality of Life Scale ${ }^{24}$ and the Positive and Negative Syndrome Scale ${ }^{25}(r=-0.39)$, 
Montgomery-Asberg Depression Rating Scale ${ }^{26}(r=-0.16)$, Subjective Well-being Under Neuroleptics Scale Short Form (SWN-Short Form $)^{27}(r=0.20)$, and Schizophrenia Objective Functioning Instrument (SOFI) ${ }^{28}(r=0.67) .{ }^{29}$ In a meta-analysis of studies examining the relationship between HRQOL and psychiatric symptoms in schizophrenia, general psychopathology was found to more strongly correlate with HRQOL than with positive or negative symptoms. ${ }^{30}$ In their interpretation of these results, the authors suggest that treatments that also target nonpsychotic symptoms may play an important role in improving HRQOL for individuals with schizophrenia. Beyond measures of clinical symptoms, another meta-analytic study found positive correlations between measures of observer-reported HRQOL and neurocognition, ${ }^{31}$ which has been shown to improve during treatment with olanzapine. ${ }^{32-35}$

The results from this study provide additional support for the use of self-reported outcomes in patients with schizophrenia. An examination of the validity of self-reported outcomes in medication-treated patients with persistent psychotic disorders living in the community found that patients were able to complete the WHOQOL-BREF with little difficulty and that the WHOQOL-BREF was highly sensitive to changes in health status. ${ }^{4}$ The sensitivity to change in health status was demonstrated by both patient-completed and case managercompleted instruments. This, combined with the current findings, suggests that patient-reported measures may play a valuable role in assessing schizophrenia outcomes. Furthermore, the addition of HRQOL measures provides for a complementary assessment of overall functioning and goes beyond the typical reporting of changes in positive, negative, and affective symptoms in clinical trials. Developing a more complete understanding of the patient's experience may facilitate treatment decisions, help improve treatment compliance, and ultimately help improve treatment outcomes. The potential utility for HRQOL assessment in clinical practice aligns well with the treatment guidelines for schizophrenia ${ }^{3}$ and may assist in furthering our understanding of psychosis. ${ }^{4}$

\section{Limitations}

This study was designed to reflect and capture usual clinical practice. However, as is the case with all nonblinded observational studies, we cannot rule out the potential influence of observer bias. Consistent with usual clinical practice, the diagnosis of schizophrenia and the determination of inadequate control on a typical antipsychotic were based on the expertise of the treating physician, rather than more robust clinical interviews or measures. Because this is a single-group study, the effects of treatment cannot be disentangled from potential improvements because of the passage of time or the use of adjunctive medications. The psychometrics of the WHOQOL-BREF have been well-established, and some research has demonstrated support for the use of patient-assessed HRQOL in schizophrenia. However, using self-report information among patients with active psychosis may increase measurement error. Patients in this study were switched from a typical antipsychotic to olanzapine, and the findings may not generalize to switches to other antipsychotics, particularly switches to typical antipsychotics in the social functioning domain of HRQOL. ${ }^{36}$

\section{Conclusion}

Statistically significant improvements in clinical symptoms and HRQOL were observed in patients from the People's Republic of China with schizophrenia. The correlations between patient-reported clinical symptoms and HRQOL measures in this study were statistically significant; however, the relationship between the two is not definitive. Assessing a patient's quality of life has the potential to add valuable clinical information that takes into account the patient's own perspective of well-being and is complementary to the assessment of clinical symptoms. Additional studies are needed to further assess the utility of including HRQOL measures in clinical trials and to develop a better understanding of their predictive value in long-term outcomes.

\section{Acknowledgments}

The authors thank Thomas Lee and Michael Stensland of Agile Outcomes Research, Inc, for their technical and writing support in the development of this manuscript.

\section{Author contributions}

All authors made substantial contributions to data generation and analysis, drafting of or critical revision to the manuscript, and approval of the final version to be published.

\section{Disclosure}

This study was sponsored by Eli Lilly and Company. WM, ZK, WY, HBX, LL, and TT are all full-time employees of Eli Lilly and Company or one of its subsidiaries, as well as minor stockholders in Eli Lilly and Company. The authors report no other conflicts of interest in this work.

\section{References}

1. Marwaha S, Johnson S. Schizophrenia and employment - a review. Soc Psychiatry Psychiatr Epidemiol. 2004;39(5):337-349.

2. Karagianis J, Novick D, Pecenak J, et al. Worldwide-Schizophrenia Outpatient Health Outcomes (W-SOHO): baseline characteristics of pan-regional observational data from more than 17,000 patients. Int $J$ Clin Pract. 2009;63(11):1578-1588. 
3. Lehman AF, Lieberman JA, Dixon LB, et al; American Psychiatric Association; Steering Committee on Practice Guidelines. Practice guideline for the treatment of patients with schizophrenia, second edition. Am J Psychiatry. 2004;161(2 Suppl):1-56.

4. Herrman H, Hawthorne G, Thomas R. Quality of life assessment in people living with psychosis. Soc Psychiatry Psychiatr Epidemiol. 2002;37(11):510-518.

5. World Health Organization. Measuring Quality of Life. Geneva: World Health Organization; 1997. Available from: http:/www.who.int/ mental_health/media/68.pdf. Accessed November 20, 2014.

6. Awad AG, Voruganti LNP. Impact of atypical antipsychotics on quality of life in patients with schizophrenia. CNS Drugs. 2004;18(13):877-893.

7. Awad AG, Voruganti LNP. Measuring quality of life in patients with schizophrenia: an update. Pharmacoeconomics. 2012;30(3):183-195.

8. Bobes J, García-Portilla P, Sáiz PA, Bascarán T, Bousoño M. Quality of life measures in schizophrenia. Eur Psychiatry. 2005;20(suppl 3): S313-S317.

9. Karow A, Naber D. Subjective well-being and quality of life under atypical antipsychotic treatment. Psychopharmacology (Berl). 2002; 162(1):3-10.

10. Boyer L, Baumstarck K, Boucekine M, Blanc J, Lançon C, Auquier P. Measuring quality of life in patients with schizophrenia: an overview. Expert Rev Pharmacoecon Outcomes Res. 2013;13(3):343-349.

11. Hunter R, Barry S. Negative symptoms and psychosocial functioning in schizophrenia: neglected but important targets for treatment. Eur Psychiatry. 2012;27(6):432-436.

12. Phillips GA, Van Brunt DL, Roychowdhury SM, Xu W, Naber D. The relationship between quality of life and clinical efficacy from a randomized trial comparing olanzapine and ziprasidone. J Clin Psychiatry. 2006;67(9):1397-1403.

13. Thwin SS, Hermes E, Lew R, et al. Assessment of the minimum clinically important difference in quality of life in schizophrenia measured by the Quality of Well-Being Scale and disease-specific measures. Psychiatry Res. 2013;209(3):291-296.

14. Wehmeier PM, Kluge M, Schacht A, Helsberg K, Schreiber W Correlation of physician and patient rated quality of life during antipsychotic treatment in outpatients with schizophrenia. Schizophr Res. 2007;91(1-3):178-186.

15. $\mathrm{Lu} \mathrm{Z,} \mathrm{Hu} \mathrm{J,} \mathrm{Chen} \mathrm{C-K,} \mathrm{et} \mathrm{al.} \mathrm{Effectiveness} \mathrm{and} \mathrm{safety} \mathrm{of} \mathrm{olanzapine}$ in the treatment of schizophrenia among Asian patients switching from conventional antipsychotics. Prog Neuropsychopharmacol Biol Psychiatry. 2007;31(1):32-40.

16. American Psychiatric Association. Diagnostic and Statistical Manual of Mental Disorders. 4th ed. Washington, DC: American Psychiatric Association; 1994.

17. World Health Organization. The ICD-10 classification of mental and behavioural disorders: diagnostic criteria for research. Geneva: World Health Organization; 1993.

18. Overall JE, Gorham DR. The Brief Psychiatric Rating Scale (BPRS): recent developments in ascertainment and scaling. Psychopharmacol Bull. 1988;24:97-99.

19. National Institute of Mental Health. Clinical Global Impressions (CGI). In: Guy W, ed. ECDEU Assessment Manual for Psychopharmacology. Bethesda: US Department of Health, Education and Welfare; 1976: 217-222.
20. World Health Organization. WHOQOL-BREF: Introduction, administration, scoring and generic version of the assessment, field trial version. Geneva, Switzerland: Programme on Mental Health; 1996.

21. Kluge M, Wehmeier PM, Dittmann RW, et al. A simple switching strategy for inadequately treated patients with schizophrenia to olanzapine: changes in psychopathology and subjective well-being. Pharmacopsychiatry. 2005;38(1):6-12.

22. Cohen J. Statistical power analysis for the behavioral sciences. Hillsdale, NJ: L. Erlbaum Associates; 1988.

23. Voruganti L, Cortese L, Owyeumi L, et al. Switching from conventional to novel antipsychotic drugs: results of a prospective naturalistic study. Schizophr Res. 2002;57(2-3):201-208.

24. Heinrichs DW, Hanlon TE, Carpenter WT Jr. The Quality of Life Scale: an instrument for rating the schizophrenic deficit syndrome. Schizophr Bull. 1984;10(3):388-398.

25. Kay SR, Fiszbein A, Opler LA. The positive and negative syndrome scale (PANSS) for schizophrenia. Schizophr Bull. 1987;13(2):261-276.

26. Montgomery SA, Asberg M. A new depression scale designed to be sensitive to change. Br J Psychiatry. 1979;134(4):382-389.

27. Naber D, Moritz S, Lambert M, et al. Improvement of schizophrenic patients' subjective well-being under atypical antipsychotic drugs. Schizophr Res. 2001;50(1-2):79-88.

28. Kleinman L, Lieberman J, Dube S, et al. Development and psychometric performance of the schizophrenia objective functioning instrument: an interviewer administered measure of function. Schizophr Res. 2009; 107(2-3):275-285.

29. Chen L, Phillips G, Johnston J, et al. The relationship, structure and profiles of schizophrenia measurements: a post-hoc analysis of the baseline measures from a randomized clinical trial. BMC Psychiatry. 2011;11(1):203.

30. Eack SM, Newhill CE. Psychiatric symptoms and quality of life in schizophrenia: a meta-analysis. Schizophr Bull. 2007;33(5):1225-1237.

31. Tolman AW, Kurtz MM. Neurocognitive predictors of objective and subjective quality of life in individuals with schizophrenia: a metaanalytic investigation. Schizophr Bull. 2012;38(2):304-315.

32. Guo X, Zhai J, Wei Q, et al; Early-stage Schizophrenia Outcome Study (ESOS) Investigators. Neurocognitive effects of first- and second-generation antipsychotic drugs in early-stage schizophrenia: a naturalistic 12-month follow-up study. Neurosci Lett. 2011;503(2) $141-146$.

33. Keefe RSE, Young CA, Rock SL, Purdon SE, Gold JM, Breier A; HGGN Study Group. One-year double-blind study of the neurocognitive efficacy of olanzapine, risperidone, and haloperidol in schizophrenia. Schizophr Res. 2006;81(1):1-15.

34. Keefe RSE, Sweeney JA, Gu H, et al. Effects of olanzapine, quetiapine, and risperidone on neurocognitive function in early psychosis: a randomized, double-blind 52-week comparison. Am J Psychiatry. 2007; 164(7):1061-1071.

35. Lindenmayer J-P, Khan A, Iskander A, Abad MT, Parker B. A randomized controlled trial of olanzapine versus haloperidol in the treatment of primary negative symptoms and neurocognitive deficits in schizophrenia. J Clin Psychiatry. 2007;68(3):368-379.

36. Fujimaki K, Takahashi T, Morinobu S. Association of typical versus atypical antipsychotics with symptoms and quality of life in schizophrenia. PLoS One. 2012;7(5):e37087.
Neuropsychiatric Disease and Treatment

\section{Publish your work in this journal}

Neuropsychiatric Disease and Treatment is an international, peerreviewed journal of clinical therapeutics and pharmacology focusing on concise rapid reporting of clinical or pre-clinical studies on a range of neuropsychiatric and neurological disorders. This journa is indexed on PubMed Central, the 'PsycINFO' database and CAS,

\section{Dovepress}

and is the official journal of The International Neuropsychiatric Association (INA). The manuscript management system is completely online and includes a very quick and fair peer-review system, which is all easy to use. Visit http://www.dovepress.com/testimonials.php to read real quotes from published authors. 